\title{
1-20 GHz distributed power amplifier based on shared artificial transmission lines
}

\author{
Ying Zhang ${ }^{1,2 a)}$, Kaixue $\mathrm{Ma}^{2}$, Hua Yang ${ }^{1}$, Yi Zhang ${ }^{1}$, \\ and Yufeng Guo ${ }^{1}$ \\ ${ }^{1}$ National and Local Joint Engineering Laboratory of RF Integration and \\ Micro-assembly Technology, Nanjing University of Posts and Telecommunications, \\ Nanjing, 210023, China \\ ${ }^{2}$ School of Physical Electronics, University of Electronic Science \\ and Technology of China, \\ Chengdu, 610054, China
}

a)zhangying@njupt.edu.cn

\begin{abstract}
A 1-20 GHz distributed power amplifier (DPA) with a novel compact structure is designed and implemented in a commercial $0.18 \mu \mathrm{m}$ CMOS technology. The proposed DPA consists of two distributed amplifiers (DAs), which have separate input artificial transmission lines (ATLs) but with their output ATLs shared to achieve high output power and efficiency in the wide frequency band. The gradually changed output ATL is used to further improve the power performance. Measurement results show that the DPA achieves $9.6 \mathrm{~dB}$ average associated gain from 1 to $20 \mathrm{GHz}$. The output power at $1-\mathrm{dB}$ output compression point $\left(O P_{1 \mathrm{~dB}}\right)$ is more than $8.2 \mathrm{dBm}$, and the peak power-added efficiency (PAE) is $9.6 \%$ with the $O P_{1 \mathrm{~dB}}$ of $12.9 \mathrm{dBm}$ at $4 \mathrm{GHz}$.
\end{abstract}

Keywords: distributed amplifier, artificial transmission line, power-added efficiency

Classification: Integrated circuits

\section{References}

[1] Z. Dai, et al:: "A new distributed parameter broadband matching method for power amplifier via real frequency technique," IEEE Trans. Microw. Theory Techn. 63 (2015) 449 (DOI: 10.1109/TMTT.2014.2385087).

[2] J. Chen and M. N. Ali: "Design and analysis of a stage-scaled distributed power amplifier," IEEE Trans. Microw. Theory Techn. 59 (2011) 1274 (DOI: 10.1109/TMTT.2011.2125985).

[3] G. Nikandish and A. Medi: "Unilateralization of MMIC distributed amplifiers," IEEE Trans. Microw. Theory Techn. 62 (2014) 3041 (DOI: 10.1109/TMTT. 2014.2361341).

[4] X. Zhou, et al:: "1 W, highly efficient, ultra-broadband non-uniform distributed power amplifier in GaN," IEEE Microw. Wireless Compon. Lett. 23 (2013) 208 (DOI: 10.1109/LMWC.2013.2250270).

[5] D.-W. Kim: "An output matching technique for a GaN distributed power amplifier MMIC using tapered drain shunt capacitors," IEEE Microw. Wireless 
Compon. Lett. 25 (2015) 603 (DOI: 10.1109/LMWC.2015.2451351).

[6] K. W. Kobayashi, et al.: “A novel $100 \mathrm{MHz}-45 \mathrm{GHz}$ input-termination-less distributed amplifier design with low-frequency low-noise and high linearity implemented with a 6 Inch $0.15 \mu \mathrm{m}$ GaN-SiC wafer process technology," IEEE J. Solid-State Circuits 51 (2016) 2017 (DOI: 10.1109/JSSC.2016.2558488).

[7] J. Kim, et al.: “A 6-18 GHz, $26 \mathrm{~W}$ GaN HEMT compact power-combined nonuniform distributed amplifier," Electron. Lett. 52 (2016) 2040 (DOI: 10.1049/ el.2016.3524).

[8] J.-C. Kao, et al:: “A novel distributed amplifier with high gain, low noise, and high output power in CMOS technology," IEEE Trans. Microw. Theory Techn. 61 (2013) 1533 (DOI: 10.1109/TMTT.2013.2247048).

[9] T.-Y. Huang, et al.: "A high-gain low-noise distributed amplifier with low DC power in $0.18-\mu \mathrm{m}$ CMOS for vital sign detection radar," IEEE MTT-S Int. Microw. Symp. (2015) 1 (DOI: 10.1109/MWSYM.2015.7166846).

[10] P. Chen, et al.: "A $22-31 \mathrm{GHz}$ distributed amplifier based on high-pass transmission lines using $0.18 \mu \mathrm{m}$ CMOS technology," IEEE Microw. Wireless Compon. Lett. 21 (2011) 160 (DOI: 10.1109/LMWC.2010.2104138).

\section{Introduction}

The rapid development of wireless and optical communications makes the total bandwidth of the commercial mobile communication technology cover a wide bandwidth more than one octave, and the low cost is expected for the base-station transceivers, which drives the high efficiency and broadband power amplifier (PA) [1]. Distributed amplifiers (DAs) provide an effective approach for extending the bandwidth and therefore are widely used in the design of ultra-wideband systems. With the process down-scaling and the cutoff frequency extending beyond $100 \mathrm{GHz}, \mathrm{CMOS}$ technology has the advantages of integration with other radio frequency (RF) and baseband circuits on the same substrate toward system on chip (SoC) as compared to GaAs PHEMT technology [2, 3] and GaN HEMT technology $[4,5,6,7]$, and it becomes promising to design wideband DA on CMOS. One major deficiency of most reported CMOS DAs $[7,8,9]$ is their low output power and efficiency due to the low breakdown voltage and the high silicon substrate loss, which impedes them to be integrated as PAs in SOC.

In this Letter, we present a novel wideband CMOS distributed power amplifier (DPA) based on shared artificial transmission lines (ATLs). The DPA consists of two ATL based DAs in a compact structure, and the gradually changed output ATL further improves the power performance. The fabricated DPA achieves more than $8.2 \mathrm{dBm}$ output power at $1-\mathrm{dB}$ output compression point $\left(O P_{1 \mathrm{~dB}}\right)$ with the poweradded efficiency (PAE) of 3.5-9.6\% in the frequency band of $1-20 \mathrm{GHz}$.

\section{Circuit design and simulation}

As shown in Fig. 1, the schematic of the proposed DPA consists of two DAs, which have separate input artificial transmission lines (ATLs) but with a shared ATL in their outputs.

According to the transmission line theory, the characteristic impedance $Z_{0}$ of the input or output ATL in DAs is given as 


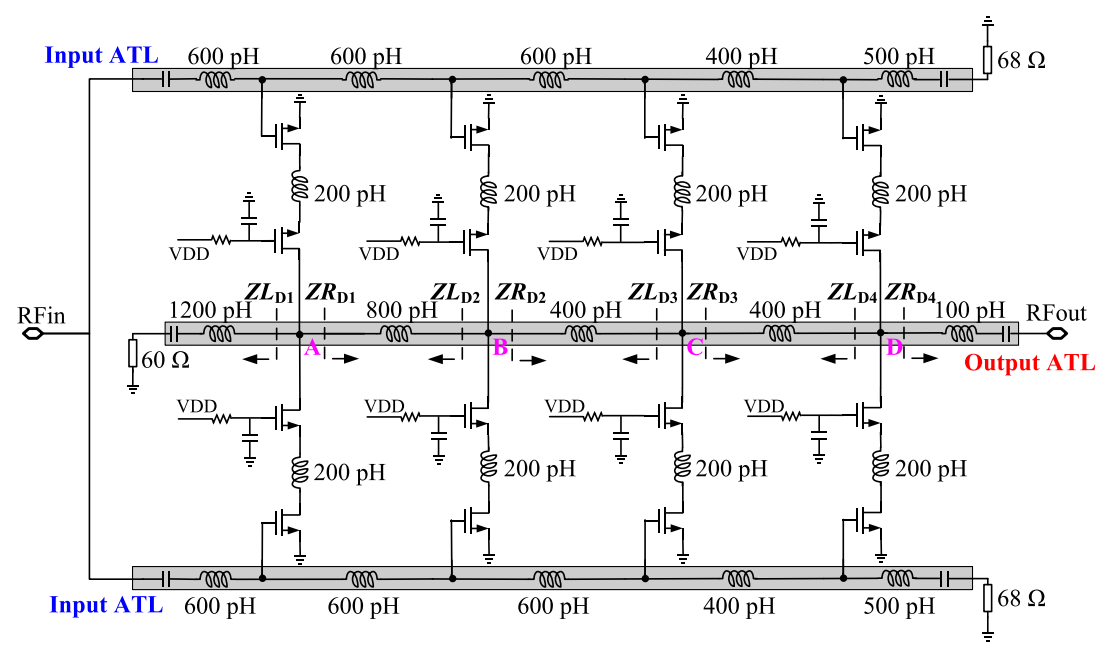

Fig. 1. Schematic diagram of proposed DPA

$$
Z_{0}=\sqrt{\frac{L_{\mathrm{g}}}{C_{\mathrm{in}}}}=\sqrt{\frac{L_{\mathrm{d}}}{C_{\mathrm{out}}}}
$$

where $C_{\mathrm{in}}$ and $C_{\mathrm{out}}$ are the input and output parasitic capacitance of gain cells, respectively; $L_{\mathrm{g}}$ and $L_{\mathrm{d}}$ are the on-chip inductor of input and output ATLs, respectively. The bandwidth of DAs is mainly restricted by the cutoff frequency $f_{\mathrm{c}}$ of input and output ATLs:

$$
f_{c}=\frac{1}{2 \pi \sqrt{L_{\mathrm{g}} C_{\mathrm{in}}}}=\frac{1}{2 \pi \sqrt{L_{\mathrm{d}} C_{\mathrm{out}}}}
$$

According to Eq. (1) and Eq. (2), the cutoff frequency $f_{\mathrm{c}}$ decreases with the increase of $C_{\text {in }}\left(C_{\text {out }}\right)$. However, the transistors in DPAs need to be large enough to provide high transconductance and output power, which results in the large parasitic capacitance, and thus a narrow bandwidth. Furthermore, $C_{\text {in }}$ is usually larger than $C_{\text {out }}$ and acts as the most important limiting factor for bandwidth. For example, the gate width of transistors in the cascode gain cell is set as $120 \mu \mathrm{m}$, and the input and output parasitic capacitance of the gain cell are presented in Fig. 2. It is apparent that the input parasitic capacitance is more than twice as large as the output one.

There are two separate input ATLs and one output ATL in the proposed DPA, as shown in Fig. 1. The equivalent capacitance at the nodes of A $\sim D$ in the output ATL is twice as large as the output parasitic capacitance of each gain cell, and therefore it releases the bandwidth constraint due to the imbalance of the input and output parasitic capacitance of gain cells. The output current at each node of $A \sim D$ is also twice as large as the output current of each gain cell, so the gate width of all transistors in Fig. 1 shrinks to $60 \mu \mathrm{m}$ to reduce the parasitic capacitance in the ATLs, while maintaining the same transconductance and output power of single gain cell in which the transistors are with $120 \mu \mathrm{m}$ gate width. Since two input ATLs are separate and connected in parallel at the input port, non-uniform input ATLs are designed to make their input impedances close to $100 \Omega$ instead of $50 \Omega$. The output power of the DPA is the accumulation of the output power of each gain cell. In Fig. $1, Z L_{\mathrm{Di}}$ and $Z R_{\mathrm{Di}}$ (the subscript $\mathrm{i}$ is from 1 to 4 ) are the impedance seen 
leftwards and rightwards at the output point of each gain cell, which are connected in parallel between the output point of each gain cell and the ground. According to Norton's theorem, more power will flow rightwards and reach the output port if $Z L_{\mathrm{Di}}$ is larger than $Z R_{\mathrm{Di}}$ for the same parallel impedance consisting of $Z L_{\mathrm{Di}}$ and $Z R_{\mathrm{Di}}$. Hence the on-chip inductors $L_{\mathrm{Di}}$ reduces from $1200 \mathrm{pH}$ to $100 \mathrm{pH}$ gradually from the left to the right output port, as shown in Fig. 1. Fig. 3 shows the corresponding impedances $Z L_{\mathrm{Di}}$ and $Z R_{\mathrm{Di}}$. It can be seen that $Z L_{\mathrm{Di}}$ (solid lines) is larger than $Z R_{\mathrm{Di}}$ (dashed lines) in the desired frequency band except some narrow frequency band, which is due to the requirement of the output impedance matching.

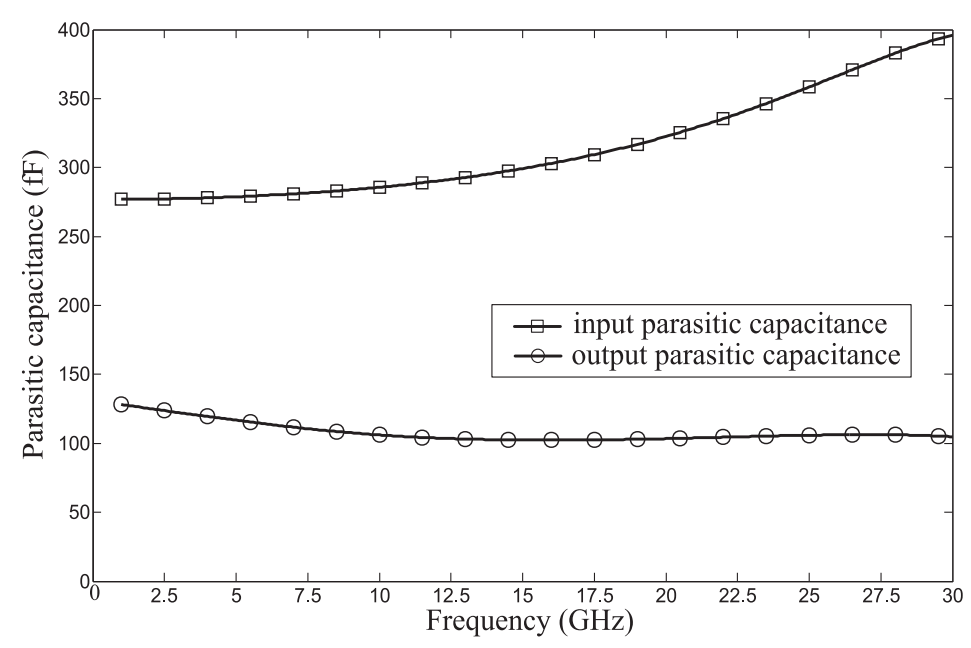

Fig. 2. Input and output parasitic capacitance of gain cell

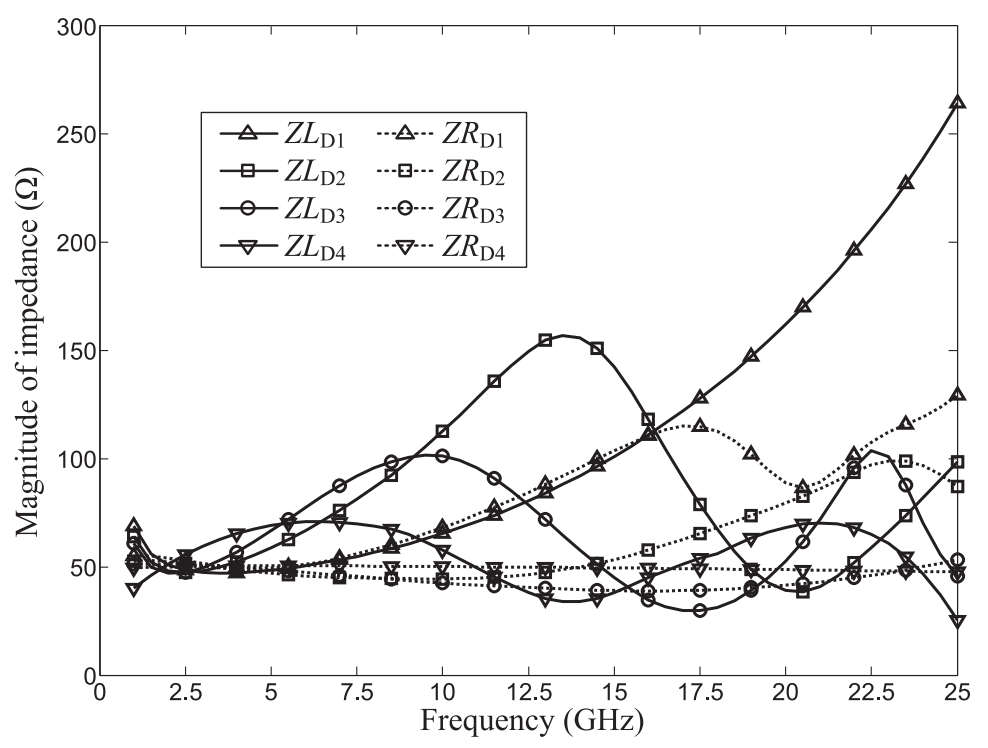

Fig. 3. Impedance at the output point of each gain cell

\section{Experimental results and discussion}

Fig. 4 is the die photograph of the designed DPA, whose size is $1.32 \mathrm{~mm} \times$ $0.77 \mathrm{~mm}$ containing probe pads. The circuit is measured via on-wafer test. 
The amplifier consumes $168 \mathrm{~mW}$ power dissipation with the supply voltage of $2.8 \mathrm{~V}$. Fig. 5 shows the measured S-parameters of the fabricated DPA. The measured average forward gain $\left(S_{21}\right)$ is $9.6 \mathrm{~dB}$ from 1 to $20 \mathrm{GHz}$ with a peak gain of $10.8 \mathrm{~dB}$ at $7.6 \mathrm{GHz}$. The input return loss $\left(S_{11}\right)$ is better than $9.9 \mathrm{~dB}$ from 1 to $21 \mathrm{GHz}$, and the output return loss $\left(S_{22}\right)$ is better than $8.3 \mathrm{~dB}$ from 1 to $25 \mathrm{GHz}$. The inaccurate inductor model and unexpected loss in the circuit leads to the acceptable discrepancy between the simulation and the measurement.

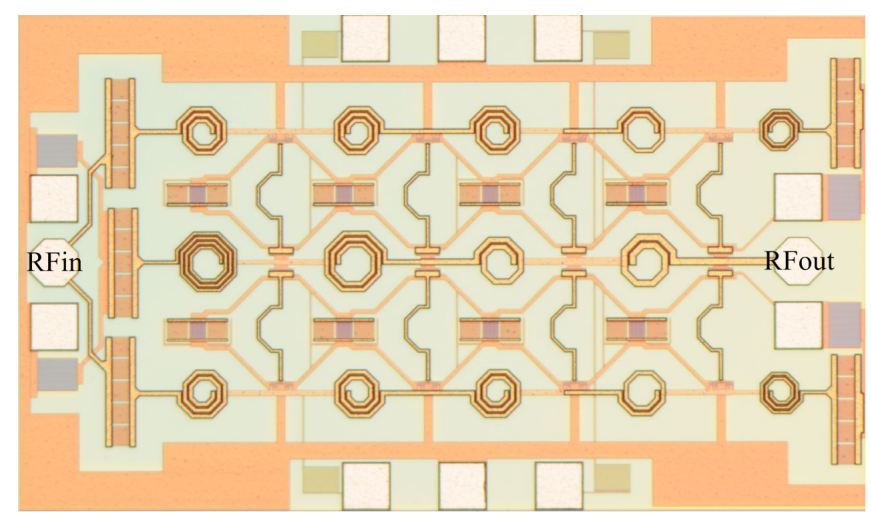

Fig. 4. Die photograph of proposed DPA (chip size: $1.32 \mathrm{~mm} \times$ $0.77 \mathrm{~mm})$

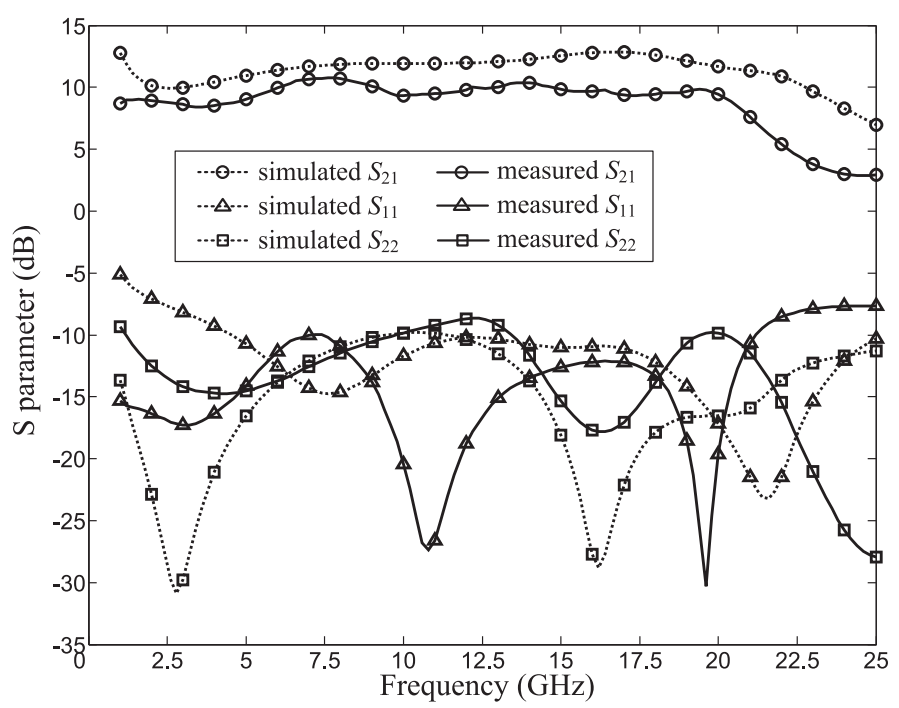

Fig. 5. Measured S-parameter of fabricated DPA at VDD $=2.8 \mathrm{~V}$.

The power measurement shows that the fabricated DPA has a good power performance, which is presented in Fig. 6. The measured $O P_{1 \mathrm{~dB}}$ is from 8.2 to $12.9 \mathrm{dBm}$ in the band of $1-20 \mathrm{GHz}$ while the corresponding PAE is from $3.5 \%$ to $9.6 \%$. Finally, Table I summarises the comparison between the fabricated DPA and the previously reported DAs. The proposed DPA exhibits high output $O P_{1 \mathrm{~dB}}$ and good PAE in the wide frequency band. 


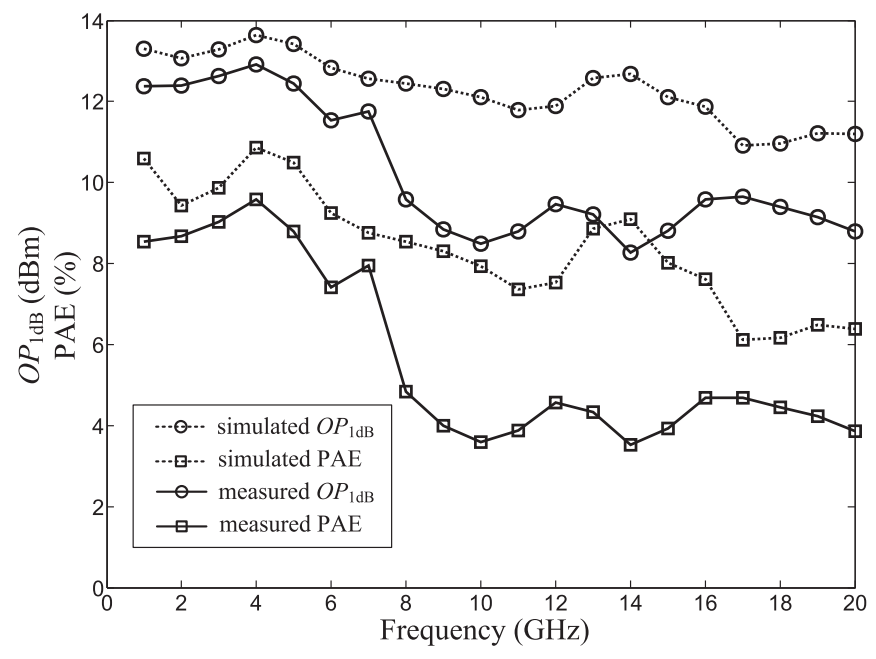

Fig. 6. Measured $O P_{1 \mathrm{~dB}}$ and PAE of fabricated DPA at VDD $=2.8 \mathrm{~V}$

Table I. Performance summary of CMOS DAs

\begin{tabular}{|c|c|c|c|c|}
\hline Refs. & {$[8]$} & {$[9]$} & {$[10]$} & This work \\
\hline Process & $0.18 \mu \mathrm{m} \mathrm{CMOS}$ & $0.18 \mu \mathrm{m} \mathrm{CMOS}$ & $0.18 \mu \mathrm{m}$ CMOS & $0.18 \mu \mathrm{m}$ CMOS \\
\hline Freq. $(\mathrm{GHz})$ & $1.5-34.2$ & $1.5-35.5$ & $22-31$ & $1-20$ \\
\hline Gain $(\mathrm{dB})$ & 24 & 25 & 6.4 & 9.6 \\
\hline VDD $(\mathrm{V})$ & 2.8 & 2.8 & 2.6 & 2.8 \\
\hline PDC $(\mathrm{mW})$ & 238 & 176.4 & 47 & 168 \\
\hline$O P_{1 \mathrm{~dB}}$ & $4.2-9 @$ & $4.6-7.4 @$ & $4.3-6.5 @$ & $8.2-12.9 @$ \\
$(\mathrm{dBm})$ & $2-30 \mathrm{GHz}^{\mathrm{a}}$ & $5-35 \mathrm{GHz}^{\mathrm{a}}$ & $22-30 \mathrm{GHz}^{\mathrm{a}}$ & $1-20 \mathrm{GHz}$ \\
\hline \multirow{2}{*}{ PAE $(\%)^{\mathrm{b}}$} & $1.1-3 @$ & $1.6-3.1 @$ & $3.9-6.5 @$ & $3.5-9.6 @$ \\
& $2-30 \mathrm{GHz}^{\mathrm{a}}$ & $5-35 \mathrm{GHz}^{\mathrm{a}}$ & $22-30 \mathrm{GHz}^{\mathrm{a}}$ & $1-20 \mathrm{GHz}$ \\
\hline Area $\left(\mathrm{mm}^{2}\right)$ & 0.83 & 0.86 & 0.17 & 1.02 \\
\hline
\end{tabular}

a This data is estimated from their papers for comparison.

b PAE is estimated at $1 \mathrm{~dB}$ gain compression point.

\section{Conclusion}

A $1-20 \mathrm{GHz}$ broadband DPA with the $O P_{1 \mathrm{~dB}}$ of more than $8.2 \mathrm{dBm}$ is implemented with a $0.18 \mu \mathrm{m}$ CMOS technology. The novel compact structure based on shared ATLs and the gradually changed output ATL improve the output power and efficiency while containing the wide operating band. The high output power and good PAE enable the DPA to work as a wideband medium-power PA.

\section{Acknowledgments}

This work is supported by the National Natural Science Foundation of China (No. 61106021), China Postdoctoral Science Foundation (No. 2015M582541) and the Natural Science Foundation of the Jiangsu Higher Education Institutions of China (No. 15KJB510020). 